\title{
Research Article \\ Computing the Moments of Order Statistics from Independent Nonidentically Distributed Exponentiated Frechet Variables
}

\author{
A. A. Jamjoom and Z. A. Al-Saiary \\ Department of Statistics, King Abdul-Aziz University, Jeddah 21589, Saudi Arabia \\ Correspondence should be addressed to A. A. Jamjoom, jamjoom-stat-6060@hotmail.com
}

Received 12 June 2011; Revised 15 October 2011; Accepted 18 October 2011

Academic Editor: Rongling Wu

Copyright (C) 2012 A. A. Jamjoom and Z. A. Al-Saiary. This is an open access article distributed under the Creative Commons Attribution License, which permits unrestricted use, distribution, and reproduction in any medium, provided the original work is properly cited.

The moments of order statistics (o.s.) arising from independent nonidentically distributed (inid) three parameter Exponentiated Frechet (EF) random variables (r.v.'s.) were computed using a theorem of Barakat and Abdelkader (2003). Two methods of integration were used to find the moments. Graphical representation of the probability density function (p.d.f.) and the cumulative distribution function (c.d.f.) of the $r$ th o.s. arising from inid r.v.'s. from this distribution. Calculations of the mean of the largest o.s. from a sample of size 2 were given for both inid and independent identically distributed (iid) r.v.'s.

\section{Introduction}

Nadarajah and Kotz [1] introduced a new lifetime model named the Exponentiated Frechet distribution EF. It is a generalization of the standard Frechet distribution (known as the extreme value distribution of type II). The EF distribution is referred to in the literature as the inverse of exponentiated Weibull distribution. The cumulative distribution function c.d.f. of the EF can be written as

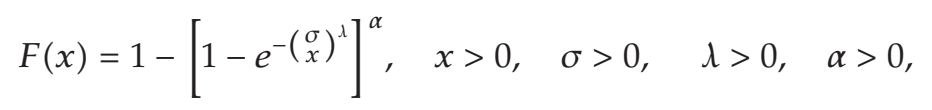

where $\alpha$ and $\lambda$ are the shape parameters and $\sigma$ is the scale parameter, respectively.

They provided a comprehensive treatment of the mathematical properties of this new distribution such as the derivation of the analytical shapes of the corresponding probability density function, the hazard rate function and provided graphical illustrations. They also 
calculated expressions for the $n$th moment, the asymptotic distribution of the extreme order statistics, investigated the variation of the skewness and kurtosis, and discussed estimation by the method of maximum likelihood.

This distribution was extensively studied by Badr [2] in a P.H.d. dessertation from several statistical points of view such as statistical properties, relation between the EF and several other distributions, statistical inferences, order statistics, record values, and associated inference.

The subject of nonidentical order statistics o.s. for EF is not discussed in the literature yet for EF distribution, which was the motivation behind this paper.

Mathematical and graphical representation of the probability density function p.d.f. and the c.d.f. of the $r$ th o.s. arising from inid EF distribution are given in Section 2.

Computation of moments of the $r$ th o.s. of inid r.v.'s. arising from inid EF using Barakat and Abdelkader [3] technique is presented in Section 3.

This technique requires that the c.d.f. of the distribution can be written in the form $F(x)=1-\lambda(x)$, which is satisfied in this distribution. This technique is referred to as Barakat Abdelkader technique (BAT).

\section{Nonidentical Order Statistics from Exponentiated Frechet Distribution}

The subject on nonidentical order statistics is discussed widely in the literature in David and Nagaraja [4]. Vaughan and Venables [5] denoted the joint p.d.f. and marginal p.d.f. of order statistics of inid random variables by means of the permanent.

Let $X_{1}, X_{2}, \ldots, X_{n}$ be independent random variables having cumulative distribution functions $F_{1}(x), F_{2}(x), \ldots, F_{n}(x)$ and probability density functions $f_{1}(x), f_{2}(x), \ldots, f_{n}(x)$, respectively. Let $X_{1: n} \leq X_{2: n} \leq \cdots \leq X_{n: n}$ denote the order statistics obtained by arranging the $n X_{i}^{\prime} s$ in increasing order of magnitude. Then the p.d.f. and the c.d.f. of the $r$ th order statistic $X_{r: n}(1 \leq r \leq n)$ can be written as

$$
f_{r: n}(x)=\frac{1}{(r-1) !(n-r) !} \sum_{p} \prod_{a=1}^{r-1} F_{i_{a}}(x) f_{i_{r}}(x) \prod_{c=r+1}^{n}\left\{1-F_{i_{c}}(x)\right\},
$$

where $\sum_{p}$ denotes the summation over all $n$ ! permutations $\left(i_{1}, i_{2}, \ldots, i_{n}\right)$ of $(1,2, \ldots n)$. Bapat and Beg [6] put it in the form of the permanent as

$$
\begin{gathered}
f_{r: n}(x)=\frac{1}{(r-1) !(n-r) !} \operatorname{per}[\underbrace{F(x)}_{r-1} \underbrace{f(x)}_{1} \underbrace{\{1-F(x)\}}_{n-r}], \\
F_{(r)^{(x)}}=\sum_{j=r}^{n} \sum_{p_{j}} \prod_{a=1}^{j} F_{i_{a}}(x) \prod_{a=j+1}^{n}\left[1-F_{i_{a}}(x)\right] .
\end{gathered}
$$

The p.d.f. and the c.d.f. of the $r$ th inid o.s. of EF distribution are displayed in Figures 1 and 2 for some selected values of the shape parameters $\lambda$ and $\alpha_{i}, i=1,2,3$ and for the scale parameter $\sigma=1$, when the sample size $n=3, r=1,2,3$. 

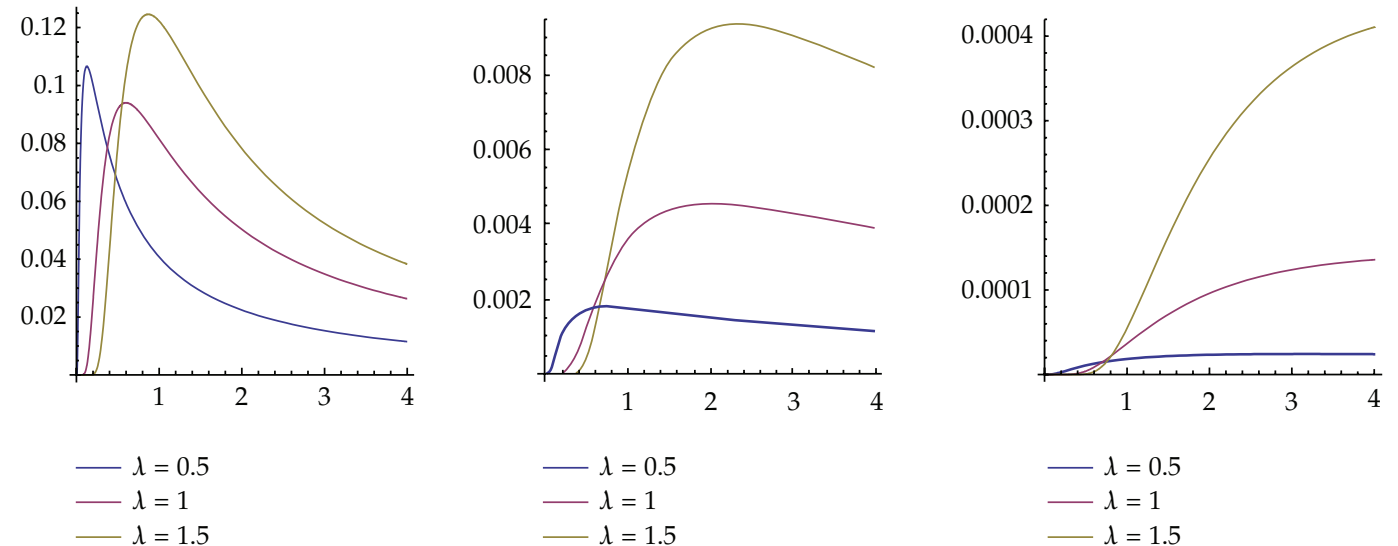

Figure 1: Graph of p.d.f. of (inid) o.s. from Ef distribution for selected values of $\alpha_{1}=0.03 ; \alpha_{2}=0.05$; $\alpha_{3}=0.07 ; \sigma=1, n=3, r=1,2,3$, respectively.

Where $\sum_{p_{j}}$ is all permutations of $\left(i_{1}, i_{2}, \ldots, i_{n}\right)$ for $(1, \ldots, n)$ which satisfy $i_{1}<i_{2}<$ $\cdots<i_{j}$ and $i_{j+1}<i_{j+2}<\cdots<i_{n}$. And using the permanent, we have

$$
F_{(r)}(x)=\sum_{i=r}^{n} \frac{1}{i !(n-i) !} \operatorname{per}\left[\begin{array}{cc}
F_{1}(x) & 1-F_{1}(x) \\
\vdots & \\
\underbrace{F_{n}(x)}_{i} & \underbrace{1-F_{n}(x)}_{n-i}
\end{array}\right], \quad-\infty<x<\infty .
$$

For EF distribution, we have

$$
\begin{gathered}
F_{i}(x)=1-\left[1-e^{-(\sigma / x)^{\lambda}}\right]^{\alpha_{i}}, \quad x>0, \quad \sigma>0, \quad \lambda>0, \quad \alpha_{i}>0, \\
f_{i}(x)=\alpha_{i} \lambda \sigma^{\lambda+1} x^{-(\lambda+1)}\left[1-e^{-(\sigma / x)^{\lambda}}\right]^{\alpha_{i}-1} e^{-(\sigma / x)^{\lambda}}, \quad x>0, \quad \sigma>0, \quad l>0, \quad \alpha_{i}>0 .
\end{gathered}
$$

\section{The Moments of the $r$ th o.s. Arising from Independent Nonidentically Distributed Exponentiated Frechet Random Variables}

Three techniques have been established in the literature to compute moments of o.s. of inid r.v.'s. see Balakrishnan [7], Barakat and Abdelkader [3], and Jamjoom and Al-Saiary [8]. Applications of the previous two methods are also found in the literature for several continuous distributions. The paper [7] established the first technique which was later referred to as differential equation technique (DET) and used it to derive recurrence relations for single and product moments of inid order statistics from the Exponential and right truncated distributions. Childs and Balakrishnan [9] applied (DET) to derive the moments of inid order statistics for logistic random variables. Mohie Elidin et al. [10] applied this method to derive the moments of inid order statistics for several distributions. 

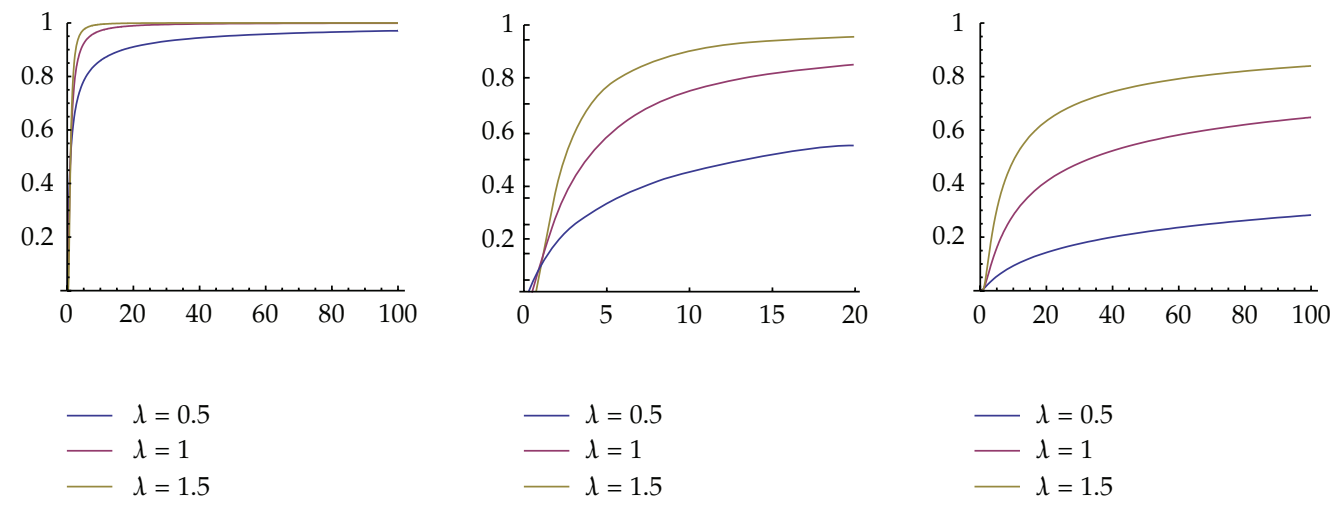

Figure 2: Graph of c.d.f. of inid o.s. from Ef distribution for selected values of $\alpha_{1}=0.03 ; \alpha_{2}=0.05 ; \alpha_{3}=0.07$; $\sigma=1, n=3, r=1,2,3$, respectively.

Barakat and Abdelkader [11] established the second technique and applied it to Weibull distribution. They generalized it in Barakat and Abdelkader [3] and applied it to several continuous distributions such as Erlang, Positive Exponential, Pareto, and Laplace distribution. This method was also used to compute the moments of inid o.s. of Gamma distribution in Abdelkader [12], Burr type XII distribution in Jamjoom [13], Beta distribution in Abdelkader [14]. Later, in Jamjoom and Al-Saiary, [15] was referred to as (BAT) and had been used to compute the moments of inid o.s. of Beta three-parameter type I distribution.

The third technique, established by Jamjoom and Al-Saiary [8], is the moment generating function technique. It depends mainly on BAT. It is referred to as (M.G.F BAT) and it was used by the same authors to compute the moments of inid o.s. for Burr type II distribution, Exponential distribution and Erlang truncated Exponential distribution.

In this section, the theorem which was established by Barakat and Abdelkader [3] will be stated without proof. Then the theorem is used to get recurrence relation for the single moments of inid o.s. arising from EF distribution.

Theorem 3.1. Let $X_{1}, X_{2}, \ldots, X_{n}$ be independent nonidentically distributed r.v.'s. The kth moment of the rth o.s. $\mu_{r: n}^{(k)}$, for $1 \leq r \leq n$ and $k=1,2, \ldots$ is given by

$$
\mu_{r: n}^{(k)}=\sum_{j=n-r+1}^{n}(-1)^{j-(n-r+1)}\left(\begin{array}{c}
j-1 \\
n-r
\end{array}\right) I_{j}(k)
$$

where

$$
I_{j}(k)=\sum_{1 \leq i_{1}<i_{2}<\cdots<i_{j} \leq n} \ldots \sum k \int_{0}^{\infty} x^{k-1} \prod_{t=1}^{j} G_{i_{t}}(x) d x, \quad j=1,2, \ldots, n,
$$

$G_{i_{t}}(x)=1-F_{i_{t}}(x)$, with $\left(i_{1}, i_{2}, \ldots, i_{n}\right)$ are permutations of $(1,2, \ldots, n)$ for which $i_{1} \leq i_{2}<\cdots<i_{n}$. The following theorem gives an explicit expression for $I_{j}(k)$ when $X_{1}, X_{2}, \ldots, X_{n}$ are inid EF r.v.'s. Two cases were considered. 
Journal of Probability and Statistics

\subsection{Case 1 (When The Shape Parameter $\alpha$ of EF Distribution Is an Integer)}

Theorem 3.2. For $1 \leq r \leq n, k=1,2, \ldots$,

$$
I_{j}(k)=\frac{k \sigma^{k} \Gamma(-k / \lambda)}{\lambda} \sum_{1 \leq i_{1}<i_{2}<\cdots<i_{j} \leq n} \sum_{m_{1}=0}^{\alpha_{i_{1}}} \cdots \sum_{m_{j}=0}^{\alpha_{i_{j}}} \xi_{i_{j}}\left(\sum_{t=1}^{j} m_{t}\right)^{k / \lambda}
$$

where

$$
\xi_{i_{j}}=\prod_{t=1}^{j} \frac{(-1)^{m_{t}} \Gamma\left(\alpha_{i_{t}}+1\right)}{m_{t} ! \Gamma\left(\alpha_{i_{t}}-m_{t}+1\right)}
$$

or

$$
I_{j}(k)=\frac{k \sigma^{k} \Gamma(-k / \lambda)}{\lambda} \sum_{1 \leq i_{1}<i_{2}<\cdots<i_{j} \leq n} \sum_{m=0} \sum_{i_{j}}\left(\begin{array}{c}
S_{i_{j}} \\
m
\end{array}\right)(-1)^{S_{i_{j}}-m}\left(S_{i_{j}}-m\right)^{k / \lambda}
$$

where

$$
S_{i_{j}}=\sum_{t=1}^{j} \alpha_{i_{t}}
$$

Proof. On applying Theorem 3.1 and using (2.4), we get

$$
I_{j}(k)=\sum_{1 \leq i_{1}<i_{2}<\cdots<i_{j} \leq n} k \int_{0}^{\infty} x^{k-1} \prod_{t=1}^{j}\left[1-e^{-(\sigma / x)^{\lambda}}\right]^{\alpha_{i t}} d x
$$

This integral converges if $\sum_{t=1}^{j} \alpha_{i_{t}}>k / \lambda$, and we used two methods of integration.

The First Method

The first method is to find this integral by expanding the expression $(1+z)^{a}$. When the exponent $(a)$ is an integer, we will use the series

$$
(b+z)^{a}=\sum_{m=0}^{a}\left(\begin{array}{c}
a \\
m
\end{array}\right) b^{a-m} z^{m},
$$

see Abramowitz and Stegun [16] page 10.

Using the Gamma notation and considering $b=1$, this series can be written as

$$
(1+z)^{a}=\sum_{m=0}^{a} \frac{\Gamma(a+1)}{\Gamma(a-m+1)} \frac{z^{m}}{m !} .
$$


Then the bracket in (3.7) can be written as

$$
\begin{aligned}
& {\left[1-e^{-(\sigma / x)^{\lambda}}\right]^{\alpha_{i_{t}}}=\sum_{m=0}^{\alpha_{i_{t}}} \frac{\Gamma\left(\alpha_{i_{t}}+1\right)}{\Gamma\left(\alpha_{i_{t}}-m+1\right)} \frac{(-1)^{m} e^{-m(\sigma / x)^{\lambda}}}{m !}} \\
& \therefore I_{j}(k)=\sum_{1 \leq i_{1}<i_{2}<\cdots<i_{j} \leq n} \sum_{0} \int_{0}^{\infty} x^{k-1} \prod_{t=1}^{j} \sum_{m=0}^{\alpha_{i_{t}}} \frac{\Gamma\left(\alpha_{i_{t}}+1\right)}{\Gamma\left(\alpha_{i_{t}}-m+1\right)} \frac{(-1)^{m} e^{-m(\sigma / x)^{l}}}{m !} d x \\
& =\sum_{1 \leq i_{1}<i_{2}<\cdots<i_{j} \leq n} k \int_{0}^{\infty} x^{k-1}\left(\sum_{m_{1}=0}^{\alpha_{i_{1}}} \frac{\Gamma\left(\alpha_{i_{1}}+1\right)}{\Gamma\left(\alpha_{i_{1}}-m_{1}+1\right)} \frac{(-1)^{m_{1}} e^{-m_{1}(\sigma / x)^{\lambda}}}{m_{1} !}\right. \\
& \left.\cdots \sum_{m_{j}=0}^{\alpha_{i_{j}}} \frac{\Gamma\left(\alpha_{i_{t}}+1\right)}{\Gamma\left(\alpha_{i_{j}}-m_{j}+1\right)} \times \frac{(-1)^{m_{j}} e^{-m_{j}(\sigma / x)^{\lambda}}}{m_{j} !}\right) d x \\
& \therefore I_{j}(k)=\sum_{1 \leq i_{1}<i_{2}<\cdots<i_{j} \leq n} k \int_{0}^{\infty} x^{k-1}\left(\sum_{m_{1}=0}^{\alpha_{i_{1}}} \cdots \sum_{m_{j}=0}^{\alpha_{i_{j}}} \frac{\Gamma\left(\alpha_{i_{1}}+1\right)}{\Gamma\left(\alpha_{i_{1}}-m_{1}+1\right)} \cdots \frac{\Gamma\left(\alpha_{i_{j}}+1\right)}{\Gamma\left(\alpha_{i_{j}}-m_{j}+1\right)}\right. \\
& \left.\times \frac{(-1)^{\sum_{t=1}^{j} m_{t}} e^{-\sum_{t=1}^{j} m_{t}(\sigma / x)^{\lambda}}}{\prod_{t=1}^{j} m_{t} !}\right) d x \\
& =\sum_{1 \leq i_{1}<i_{2}<\cdots<i_{j} \leq n} k \sum_{m_{1}=0}^{\alpha_{i_{1}}} \cdots \sum_{m_{j}=0}^{\alpha_{i_{j}}}\left(\prod_{t=1}^{j} \frac{\Gamma\left(\alpha_{i_{t}}+1\right)}{\Gamma\left(\alpha_{i_{t}}-m_{t}+1\right)} \frac{(-1)^{m_{t}}}{m_{t} !}\right. \\
& \left.\times \int_{0}^{\infty} x^{k-1} e^{-\sum_{t=1}^{j} m_{t}(\sigma / x)^{\lambda}} d x\right) \\
& =\sum_{1 \leq i_{1}<i_{2}<\cdots<i_{j} \leq n} k \sum_{m_{1}=0}^{\alpha_{i_{1}}} \cdots \sum_{m_{j}=0}^{\alpha_{i_{j}}}\left(\prod_{t=1}^{j} \frac{(-1)^{m_{t}}}{m_{t} !} \frac{\Gamma\left(\alpha_{i_{t}}+1\right)}{\Gamma\left(\alpha_{i_{t}}-m_{t}+1\right)}\right. \\
& \left.\times \int_{0}^{\infty} x^{k-1} e^{-\sum_{t=1}^{j} m_{t}(\sigma / x)^{\lambda}} d x\right) .
\end{aligned}
$$

Substituting

$$
\begin{aligned}
y & =\sum_{t=1}^{j} m_{t}\left(\frac{\sigma}{x}\right)^{\lambda} \\
\therefore x & =\sigma\left(\sum_{t=1}^{j} m_{t}\right)^{1 / \lambda} y^{-1 / \lambda}
\end{aligned}
$$


Journal of Probability and Statistics

$$
\begin{aligned}
d x & =\frac{\sigma}{\lambda}\left(\sum_{t=1}^{j} m_{t}\right)^{1 / \lambda} y^{-(1 / \lambda)-1} d y \\
\therefore \int_{0}^{\infty} x^{k-1} e^{-\sum_{t=1}^{j} m_{t}(\sigma / x)^{\lambda}} d x & =\sigma^{k}\left(\sum_{t=1}^{j} m_{t}\right)^{k / \lambda} \int_{0}^{\infty} y^{-(k / \lambda)-1} e^{-y} d y \\
& =\frac{\sigma^{k}}{\lambda}\left(\sum_{t=1}^{j} m_{t}\right)^{k / \lambda} \Gamma\left(-\frac{k}{\lambda}\right) ; \lambda>0, k=1,2, \ldots, \frac{k}{\lambda} \neq 0,1,2, \ldots
\end{aligned}
$$

Making some arrangements, we get (3.3).

The Second Method of Integration is Given by Using the Transformation

$$
y=e^{-(\sigma / x)^{\lambda}}
$$

in (3.7)

$$
\therefore x=\sigma\left(\ln \frac{1}{y}\right)^{-1 / \lambda}
$$

Then

$$
\begin{gathered}
d x=-\frac{\sigma}{\lambda}\left(\ln \frac{1}{y}\right)^{-(1 / \lambda)-1} y^{-1} d y \\
\therefore \int_{0}^{\infty} x^{k-1} \prod_{t=1}^{j}\left[1-e^{-(\sigma / x)^{\lambda}}\right]^{\alpha_{i_{t}}} d x=\int_{0}^{\infty} x^{k-1}\left[1-e^{-(\sigma / x)^{\lambda}}\right]^{S_{i_{j}}} d x,
\end{gathered}
$$

where

$$
\begin{aligned}
S_{i_{j}} & =\sum_{t=1}^{j} \alpha_{i_{t}} \\
& =\frac{\sigma^{k}}{\lambda} \int_{0}^{1}\left(\ln \frac{1}{y}\right)^{-(k / \lambda)-1}[1-y]^{S_{i_{j}}} y^{-1} d y \\
& =\frac{\sigma^{k}}{\lambda} \sum_{m=0}^{S_{i_{j}}}\left(\begin{array}{c}
S_{i_{j}} \\
m
\end{array}\right)(-1)^{S_{i_{j}}-m} \int_{0}^{1}\left(\ln \frac{1}{y}\right)^{-(k / \lambda)-1} y^{S_{i_{j}}-m-1} d y .
\end{aligned}
$$

Using

$$
\int_{0}^{1}\left(\ln \frac{1}{y}\right)^{\theta-1} y^{\nu-1} d y=\frac{1}{v^{\theta}} \Gamma(\theta)
$$


Table 1: $I_{j}(k)$ using (3.3) when $n=3$.

\begin{tabular}{|c|c|}
\hline j & $I_{j}(k)$ \\
\hline 1 & $\begin{aligned} I_{1}(k)=\frac{k \sigma^{k} \Gamma(-k / \lambda)}{\lambda}\left(\sum_{m_{1}=0}^{\alpha_{1}} \frac{(-1)^{m_{1}} \Gamma\left(\alpha_{1}+1\right)}{m_{1} ! \Gamma\left(\alpha_{1}-m_{1}+1\right)}\left(m_{1}\right)^{k / \lambda}+\sum_{m_{1}=0}^{\alpha_{2}} \frac{(-1)^{m_{1}} \Gamma\left(\alpha_{2}+1\right)}{m_{1} ! \Gamma\left(\alpha_{2}-m_{1}+1\right)}\left(m_{1}\right)^{k / \lambda}\right. \\
\left.\quad+\sum_{m_{1}=0}^{\alpha_{3}} \frac{(-1)^{m_{1}} \Gamma\left(\alpha_{3}+1\right)}{m_{1} ! \Gamma\left(\alpha_{3}-m_{1}+1\right)}\left(m_{1}\right)^{k / \lambda}\right)\end{aligned}$ \\
\hline 2 & $\begin{aligned} I_{2}(k)=\frac{k \sigma^{k} \Gamma(-k / \lambda)}{\lambda}( & \sum_{m_{1}=0}^{\alpha_{1}} \sum_{m_{2}=0}^{\alpha_{2}} \frac{(-1)^{m_{1}+m_{2}} \Gamma\left(\alpha_{1}+1\right) \Gamma\left(\alpha_{2}+1\right)}{m_{1} ! m_{2} ! \Gamma\left(\alpha_{1}-m_{1}+1\right) \Gamma\left(\alpha_{2}-m_{2}+1\right)}\left(m_{1}+m_{2}\right)^{k / \lambda} \\
& +\sum_{m_{1}=0}^{\alpha_{1}} \sum_{m_{2}=0}^{\alpha_{3}} \frac{(-1)^{m_{1}+m_{2}} \Gamma\left(\alpha_{1}+1\right) \Gamma\left(\alpha_{3}+1\right)}{m_{1} ! m_{2} ! \Gamma\left(\alpha_{1}-m_{1}+1\right) \Gamma\left(\alpha_{3}-m_{2}+1\right)}\left(m_{1}+m_{2}\right)^{k / \lambda} \\
& +\sum_{m_{1}=0}^{\alpha_{2}} \sum_{m_{2}=0}^{\alpha_{3}} \frac{(-1)^{m_{1}+m_{2}} \Gamma\left(\alpha_{2}+1\right) \Gamma\left(\alpha_{3}+1\right)}{m_{1} ! m_{2} ! \Gamma\left(\alpha_{2}-m_{1}+1\right) \Gamma\left(\alpha_{3}-m_{2}+1\right)}\end{aligned}$ \\
\hline & $\left.\times\left(m_{1}+m_{2}\right)^{k / \lambda}\right)$ \\
\hline 3 & $\begin{aligned} I_{3}(k)=\frac{k \sigma^{k} \Gamma(-k / \lambda)}{\lambda}\left(\sum_{m_{1}=0}^{\alpha_{1}} \sum_{m_{2}=0}^{\alpha_{2}} \sum_{m_{3}=0}^{\alpha_{3}} \frac{(-1)^{m_{1}+m_{2}+m_{3}} \Gamma\left(\alpha_{1}+1\right) \Gamma\left(\alpha_{2}+1\right) \Gamma\left(\alpha_{3}+1\right)}{m_{1} ! m_{2} ! m_{3} ! \Gamma\left(\alpha_{1}-m_{1}+1\right) \Gamma\left(\alpha_{2}-m_{2}+1\right) \Gamma\left(\alpha_{2}-m_{2}+1\right)}\right. \\
\left.\quad \times\left(m_{1}+m_{2}+m_{3}\right)^{k / \lambda}\right)\end{aligned}$ \\
\hline
\end{tabular}

Table 2: $I_{j}(k)$ using (3.5) when $n=3$.

\begin{tabular}{|c|c|}
\hline$j$ & $I_{j}(k)$ \\
\hline 1 & $I_{1}(k)=\frac{k \sigma^{k} \Gamma(-(k / \lambda))}{\lambda} \sum_{i=1}^{n} \sum_{m=0}^{\alpha_{i}}\left(\begin{array}{c}\alpha_{i} \\
m\end{array}\right)(-1)^{\alpha_{i}-m}\left(\alpha_{i}-m\right)^{k / \lambda}$ \\
\hline & $I_{2}(k)=\frac{k \sigma^{k} \Gamma(-k / \lambda)}{\lambda}\left(\sum_{m=0}^{\alpha_{1}+\alpha_{2}}\left(\begin{array}{c}\alpha_{1}+\alpha_{2} \\
m\end{array}\right)(-1)^{\alpha_{1}+\alpha_{2}-m}\left(\alpha_{1}+\alpha_{2}-m\right)^{k / \lambda}\right.$ \\
\hline 2 & $\begin{aligned} & +\sum_{m=0}^{\alpha_{1}+\alpha_{3}}\left(\begin{array}{c}\alpha_{1}+\alpha_{3} \\
m\end{array}\right)(-1)^{\alpha_{1}+\alpha_{3}-m}\left(\alpha_{1}+\alpha_{3}-m\right)^{k / \lambda} \\
& \left.+\sum_{m=0}^{\alpha_{2}+\alpha_{3}}\left(\begin{array}{c}\alpha_{2}+\alpha_{3} \\
m\end{array}\right)(-1)^{\alpha_{2}+\alpha_{3}-m}\left(\alpha_{2}+\alpha_{3}-m\right)^{k / \lambda}\right)\end{aligned}$ \\
\hline 3 & $\frac{k \sigma^{k} \Gamma(-k / \lambda)}{\lambda}\left(\sum_{m=0}^{\alpha_{1}+\alpha_{2}+\alpha_{3}}\left(\underset{m}{\alpha_{1}+\alpha_{2}+\alpha_{3}}\right)(-1)^{\alpha_{1}+\alpha_{2}+\alpha_{3}-m}\left(\alpha_{1}+\alpha_{2}+\alpha_{3}-m\right)^{k / \lambda}\right)^{\prime}$ \\
\hline
\end{tabular}

see (4.272.6) in Gradshteyn and Ryzhik [17]

$$
\therefore \int_{0}^{1}\left(\ln \frac{1}{y}\right)^{-(k / \lambda)-1} y^{S_{i_{j}}-m-1} d y=\frac{1}{\left(S_{i_{j}}-m\right)^{-k / \lambda}} \Gamma\left(-\frac{k}{\lambda}\right) ; \quad k \neq 0, \lambda, 2 \lambda, 3 \lambda, \ldots
$$

Substituting (3.17) in (3.15) and making some arrangements, we get (3.5).

Tables 1 and 2 give the values of $I j$ in (3.3) and (3.5), for $j=1,2,3$ and sample size $n=3$. 
Journal of Probability and Statistics

Corollary 3.3. For inid EF r.v.'s, when $j=1$, (3.3) becomes

$$
I_{1}(k)=\frac{k \sigma^{k} \Gamma(-k / \lambda)}{\lambda} \sum_{i=1}^{n} \sum_{r=1}^{\alpha_{i}}(-1)^{r} \alpha_{i}\left(\alpha_{i}-1\right) \cdots\left(\alpha_{i}-(r-1)\right) \frac{r^{k / \lambda}}{r !}
$$

Proof. One has

$$
\begin{aligned}
I_{1}(k)= & \frac{k \sigma^{k} \Gamma(-k / \lambda)}{\lambda} \sum_{i=1}^{n}\left(\sum_{m_{1}=0}^{\alpha_{i}} \xi_{i} m_{t}{ }^{k / \lambda}\right) \\
= & \frac{k \sigma^{k} \Gamma(-k / \lambda)}{\lambda} \sum_{i=1}^{n}\left(\sum_{m_{1}=0}^{\alpha_{i}} \frac{(-1)^{m_{1}} \Gamma\left(\alpha_{i}+1\right)}{m_{1} ! \Gamma\left(\alpha_{i}-m_{1}+1\right)} m_{t}{ }^{k / \lambda}\right) \\
= & \frac{k \sigma^{k} \Gamma(-k / \lambda)}{\lambda} \\
& \times \sum_{i=1}^{n}\left(0-\frac{\Gamma\left(\alpha_{i}+1\right)}{\Gamma\left(\alpha_{i}\right)}+\frac{\Gamma\left(\alpha_{i}+1\right)}{2 \Gamma\left(\alpha_{i}-1\right)} 2^{k / \lambda}-\frac{\Gamma\left(\alpha_{i}+1\right)}{3 ! \Gamma\left(\alpha_{i} s-2\right)} 3^{k / \lambda}+\frac{\Gamma\left(\alpha_{i}+1\right)}{4 ! \Gamma\left(\alpha_{i}-2\right)} 4^{k / \lambda}\right. \\
& \left.\quad+\cdots+\frac{(-1)^{\alpha_{i}} \Gamma\left(\alpha_{i}+1\right)}{\alpha_{i} ! \Gamma(1)} \alpha_{i}{ }^{k / \lambda}\right) \\
= & \frac{k \sigma^{k} \Gamma(-k / \lambda)}{\lambda} \\
& \times \sum_{i=1}^{n}\left(-\alpha_{i}+\frac{\alpha_{i}\left(\alpha_{i}-1\right)}{2} 2^{k / \lambda}-\frac{\alpha_{i}\left(\alpha_{i}-1\right)\left(\alpha_{i}-2\right)}{3 !} 3^{k / \lambda}+\frac{\alpha_{i}\left(\alpha_{i}-1\right)\left(\alpha_{i}-2\right)\left(\alpha_{i}-3\right)}{4 !} 4^{k / \lambda}\right. \\
\left.\quad+\cdots+(-1)^{\alpha_{i}} \alpha_{i}{ }^{k / \lambda}\right) & \frac{k \sigma^{k} \Gamma(-k / \lambda)}{\lambda} \sum_{i=1}^{n}\left(\sum_{r=1}^{\alpha}(-1)^{r}+\alpha_{i}\left(\alpha_{i}-1\right)+\cdots+\left(\alpha_{i}-(r-1) \frac{r^{k / \lambda}}{r !}\right)\right) .
\end{aligned}
$$

The iid case can be deduced from Theorem 3.2. The result will be stated in the next corollary.

Corollary 3.4. For the case of a sample of $n$ iid r.v.'s having EF distribution, the $I_{j}(k)$ in Theorem 3.2 simply reduces to

$$
I_{j}(k)=\frac{k \sigma^{k} \Gamma(-k / \lambda)}{\lambda}\left(\begin{array}{l}
n \\
j
\end{array}\right) \sum_{m_{1}=0}^{\alpha} \cdots \sum_{m_{j}=0}^{\alpha} \xi_{i_{j}}\left(\sum_{t=1}^{j} m_{t}\right)^{k / \lambda}
$$

where

$$
\xi_{j}=\prod_{t=1}^{j} \frac{(-1)^{m_{t}} \Gamma(\alpha+1)}{m_{t} ! \Gamma\left(\alpha-m_{t}+1\right)}
$$


Table 3: The moments $\mu_{2: 2}$ arising from iid Exponentiated Frechet r.v.'s. using (3.3) or (3.5).

\begin{tabular}{lcccc}
\hline$\alpha$ & 2 & 3 & 4 & 5 \\
\hline$\mu_{2: 2}$ & 1.2915 & 1.03527 & 0.916892 & 0.84626 \\
\hline
\end{tabular}

or

$$
I_{j}(k)=\frac{k \sigma^{k} \Gamma(-k / \lambda)}{\lambda}\left(\begin{array}{c}
n \\
j
\end{array}\right) \sum_{m=0}^{S_{j}}\left(\begin{array}{l}
S_{j} \\
m
\end{array}\right)(-1)^{S_{j}-m}\left(S_{j}-m\right)^{k / \lambda}
$$

where

$$
S_{j}=j \alpha
$$

Corollary 3.5. For iid EF r.v.'s, when $j=1$, (3.18) becomes

$$
I_{1}(k)=\frac{k n \sigma^{k} \Gamma(-k / \lambda)}{\lambda} \sum_{r=1}^{\alpha}(-1)^{r} \alpha(\alpha-1) \cdots(\alpha-(r-1)) \frac{r^{k / \lambda}}{r !}
$$

\section{Numerical Applications}

The following examples are computed when $k=1$.

Example 3.6. Let $n=2$ and $\alpha=2,3,4$, and 5. Table 3 shows the results of the calculations.

For example, when $\alpha=3, \sigma=1, \lambda=2$,

$$
\begin{aligned}
\mu_{2: 2} & =I_{1}-I_{2} \\
I_{1} & =\frac{2 \Gamma(-1 / 2)}{2} \sum_{r=1}^{3}(-1)^{r} \alpha(\alpha-1) \cdots(\alpha-(r-1)) \frac{r^{k / \lambda}}{r !} \\
& =\sqrt{\pi}\left(-\alpha+\frac{\sqrt{2} \alpha(\alpha-1)}{2}-\frac{\sqrt{2} \alpha(\alpha-1)(\alpha-2)}{6}\right) \\
& =1.73491 \\
I_{2} & =\frac{\Gamma(-1 / 2)}{2}\left(\begin{array}{l}
2 \\
2
\end{array}\right) \sum_{m_{1}=0}^{3} \sum_{m_{2}=0}^{3} \prod_{t=1}^{2} \frac{(-1)^{t} \Gamma(\alpha+1)}{m_{j} ! \Gamma\left(\alpha-m_{j}+1\right)}\left(\sum_{t=1}^{2} m_{t}\right)^{1 / 2} \\
& =0.699642 \\
\mu_{2: 2} & =1.73491-0.699642 \\
& =1.03527 .
\end{aligned}
$$

Example 3.7. Setting $n=2, \sigma=1, \lambda=2$, and $\alpha_{1}=1(1) 5, \alpha_{2}=1(1) 5$ in Theorems 3.1 and 3.2, we get the results of the calculations in Table 4 . 
Table 4: The moments $\mu_{2: 2}$ arising from inid Exponentiated Frechet r.v.'s. using (3.3) or (3.5).

\begin{tabular}{cccccc}
\hline$\alpha_{2}$ & \multicolumn{5}{c}{$\alpha_{1}$} \\
\\
\hline 1 & 1 & 2 & 3 & 4 & 5 \\
2 & 2.50663 & 1.94328 & 1.85485 & 1.82294 & 1.80739 \\
3 & 1.94328 & 1.2915 & 1.17116 & 1.1237 & 1.09925 \\
4 & 1.85485 & 1.17116 & 1.03527 & 0.978905 & 0.948808 \\
5 & 1.82294 & 1.1237 & 0.978905 & 0.916892 & 0.882953 \\
\hline
\end{tabular}

For example, when $\alpha_{1}=2, \alpha_{2}=3$

$$
\begin{aligned}
\mu_{2: 2}= & I_{1}-I_{2}, \\
I_{1}= & \frac{\Gamma(-1 / 2)}{\lambda} \sum_{i=1}^{2} \sum_{r=1}^{\infty}(-1)^{r} \alpha_{i}\left(\alpha_{i}-1\right) \cdots\left(\alpha_{i}-(r-1)\right) \frac{r^{k / \lambda}}{r !} \\
= & \frac{\Gamma(-1 / 2)}{\lambda}\left(\sum_{r=1}^{\alpha_{1}}(-1)^{r} \alpha_{1}\left(\alpha_{1}-1\right) \cdots\left(\alpha_{1}-(r-1)\right) \frac{r^{k / \lambda}}{r !}+\sum_{r=1}^{\alpha_{2}}(-1)^{r} \alpha_{2}\left(\alpha_{2}-1\right)\right. \\
& \left.\cdots\left(\alpha_{2}-(r-1)\right) \frac{r^{k / \lambda}}{r !}\right) \\
= & 1.90574 \\
I_{2}= & \frac{\Gamma(-1 / 2)}{2} \sum_{m_{1}=0}^{2} \sum_{m_{2}=0}^{3} \frac{(-1)^{m_{1}+m_{2}} \Gamma\left(\alpha_{1}+1\right) \Gamma\left(\alpha_{2}+1\right)}{m_{t} ! m ! \Gamma\left(\alpha_{1}-m_{1}+1\right) \Gamma\left(\alpha_{2}-m_{2}+1\right)}\left(m_{1}+m_{2}\right)^{1 / 2} \\
= & 0.734577 \\
\mu_{2: 2}= & 1.90574-0.734577 \\
= & s 1.17117 .
\end{aligned}
$$

\subsection{Case 2 (When the Shape Parameter $\alpha$ of EF Is Noninteger)}

When $\alpha$ is noninteger, the expansion of $(1+z)^{a}$ is then written as

$$
(1+z)^{a}=\sum_{m=0}^{\infty}\left(\begin{array}{c}
a \\
m
\end{array}\right) z^{m}, \quad-1<z<1
$$

see Abramowitz and Stegun [16] page 14. Using gamma notation, (3.27) can be written as

$$
(1+z)^{a}=\sum_{m=0}^{\infty} \frac{\Gamma(a+1)}{\Gamma(a-m+1)} \frac{z^{m}}{m !}, \quad-1<z<1 .
$$


Equations (3.3) and (3.5) become

$$
I_{j}(k)=\frac{k \sigma^{k} \Gamma(-k / \lambda)}{\lambda} \sum_{1 \leq i_{1}<i_{2}<\cdots<i_{j} \leq n} \sum_{m_{1}=0}^{\infty} \cdots \sum_{m_{j}=0}^{\infty} \xi_{i_{j}}\left(\sum_{t=1}^{j} m_{t}\right)^{k / \lambda}
$$

where

$$
\xi_{i j}=\prod_{t=1}^{j} \frac{(-1)^{m_{t}} \Gamma\left(\alpha_{i_{t}}+1\right)}{m_{t} ! \Gamma\left(\alpha_{i_{t}}-m_{t}+1\right)}
$$

or

$$
I_{j}(k)=\frac{k \sigma^{k} \Gamma(-k / \lambda)}{\lambda} \sum_{1 \leq i_{1}<i_{2}<\cdots<i_{j} \leq n} \sum_{m=0}^{\infty}\left(\begin{array}{c}
S_{i_{j}} \\
m
\end{array}\right)(-1)^{S_{i_{j}}-m}\left(S_{i_{j}}-m\right)^{k / \lambda},
$$

where

$$
S_{i_{j}}=\sum_{t=1}^{j} \alpha_{i_{t}}
$$

Corollary 3.8. For inid EF r.v.'s, when $j=1$, (3.29) becomes

$$
I_{1}(k)=\frac{k \sigma^{k} \Gamma(-k / \lambda)}{\lambda} \sum_{i=1}^{n} \sum_{r=1}^{\infty}(-1)^{r} \alpha_{i}\left(\alpha_{i}-1\right) \cdots\left(\alpha_{i}-(r-1)\right) \frac{r^{k / \lambda}}{r !} .
$$

The iid case can be deduced from (3.29). The result will be stated in the next corollary.

Corollary 3.9. For a sample of iid r.v.'s having EF distribution, the $I_{j}(k)$ in (3.29) simply reduces to

$$
I_{j}(k)=\frac{k \sigma^{k} \Gamma(-k / \lambda)}{\lambda}\left(\begin{array}{c}
n \\
j
\end{array}\right) \sum_{m_{1}=0}^{\infty} \cdots \sum_{m_{j}=0}^{\infty} \xi_{i_{j}}\left(\sum_{t=1}^{j} m_{t}\right)^{k / \lambda},
$$

where

$$
\xi_{j}=\prod_{t=1}^{j} \frac{(-1)^{m_{t}} \Gamma(\alpha+1)}{m_{t} ! \Gamma\left(\alpha-m_{t}+1\right)}
$$

or

$$
I_{j}(k)=\frac{k \sigma^{k} \Gamma(-k / \lambda)}{\lambda}\left(\begin{array}{c}
n \\
j
\end{array}\right) \sum_{m=0}^{\infty}\left(\begin{array}{l}
S_{j} \\
m
\end{array}\right)(-1)^{S_{j}-m}\left(S_{j}-m\right)^{k / \lambda},
$$


Table 5: The moments $\mu_{2: 2}$ arising from IID Exponentiated Frechet r.v.'s. using (3.29) or (3.31).

\begin{tabular}{lcccc}
\hline$\alpha$ & 1.5 & 2.5 & 3.5 & 4.5 \\
\hline$\mu_{2: 2}$ & 2.68469 & 0.666308 & 1.30236 & 0.637316 \\
\hline
\end{tabular}

where

$$
S_{j}=j \alpha .
$$

Corollary 3.10. For iid EF r.v.'s, when $j=1$, (3.33) becomes

$$
I_{1}(k) \frac{k n \sigma^{k} \Gamma(-k / \lambda)}{\lambda} \sum_{r=1}^{\infty}(-1)^{r} \alpha(\alpha-1) \cdots(\alpha-(r-1)) \frac{r^{k / \lambda}}{r !} .
$$

Example 3.11. Let $n=2$ and $\alpha=1.5,2.5,3.5,4.5$. Table 5 shows the results of calculations.

Remark 3.12. Calculations in Example 3.11 were done following the method of Abdelkader [12]. The upper limit of the sum $(\infty)$ is taken up to $[\alpha]$, where [.] is the usual greatest integer function.

Remark 3.13. All figures and tables in this paper had been accomplished by Mathematica 7.0.

\section{References}

[1] S. Nadarajah and S. Kotz, "The exponentiated frechet distribution," 2003, http:/ /interstat.statjournals .net/YEAR/2003/abstracts/0312001.php.

[2] M. Badr, Studying the exponentiated frechet distribution, Ph.D. thesis, Department of Statistics, King Abdelazeez University, Jeddah, Saudi Arabia, 2010.

[3] H. M. Barakat and Y. H. Abdelkader, "Computing the moments of order statistics from nonidentical random variables," Statistical Methods E Applications, vol. 13, no. 1, pp. 15-26, 2004.

[4] H. A. David and H. N. Nagaraja, Order Statistics, John Wiley \& Sons, New York, NY, USA, 3rd edition, 2003.

[5] R. J. Vaughan and W. N. Venables, "Permanent expressions for order statistic densities," Journal of the Royal Statistical Society B, vol. 34, pp. 308-310, 1972.

[6] R. B. Bapat and M. I. Beg, "Order statistics for nonidentically distributed variables and permanents," Sankhyā A, vol. 51, no. 1, pp. 79-93, 1989.

[7] N. Balakrishnan, "Order statistics from non-identical exponential random variables and some applications," Computational Statistics \& Data Analysis, vol. 18, no. 2, pp. 203-253, 1994.

[8] A. A. Jamjoom and Z. A. AL-Saiary, "Moment Generating Function technique for moments of order statistics from nonidentically distributed random variables," International Journal of Statistics and Systems, vol. 6, no. 2, pp. 177-188, 2011.

[9] A. Childs and N. Balakrishnan, "Relations for order statistics from non-identical logistic random variables and assessment of the effect of multiple outliers on the bias of linear estimators," Journal of Statistical Planning and Inference, vol. 136, no. 7, pp. 2227-2253, 2006.

[10] M. Mohie Elidin, M. Mahmoud, M. Moshref, and M. Mohmed, On Independent and Nonidentical Order Statistics and Associated Inference, Department of Mathematics, Al-Azhar University, Cairo, Egypt, 2007.

[11] H. M. Barakat and Y. H. Abdelkader, "Computing the moments of order statistics from nonidentically distributed Weibull variables," Journal of Computational and Applied Mathematics, vol. 117, no. 1, pp. 8590, 2000. 
[12] Y. H. Abdelkader, "Computing the moments of order statistics from nonidentically distributed gamma variable with applications," International Journal of Mathematics, Game Theory, and Algebra, vol. 14, no. 1, pp. 1-8, 2004.

[13] A. A. Jamjoom, "Computing the moments of order statistics from independent non-identically distributed Burr type XII random variables," Journal of Mathematics and Statistics, vol. 2, no. 3, pp. 432438, 2006.

[14] Y. H. Abdelkader, "Computing the moments of order statistics from independent nonidentically distributed Beta random variables," Statistical Papers, vol. 49, pp. 136-149, 2008.

[15] A. A. Jamjoom and Z. A. AL-Saiary, "Computing the moments of order statistics from independent nonidentically distributed Beta type I random variables," Journal of Mthematics and Statistics, vol. 3, 2010.

[16] M. Abramowitz and I. Stegun, Handbook of Mathematical Functions with Formulas, Graphs, and Mathematical Tables, Dover, New York, NY, USA, 1972.

[17] I. S. Gradshteyn and I. M. Ryzhik, Table of Integrals, Series, and Products, Academic Press, New York, NY, USA, 1980. 


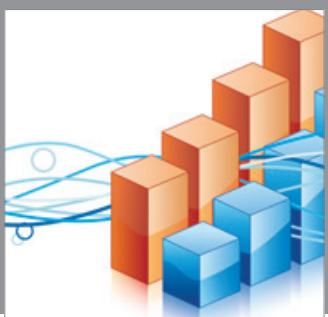

Advances in

Operations Research

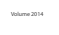

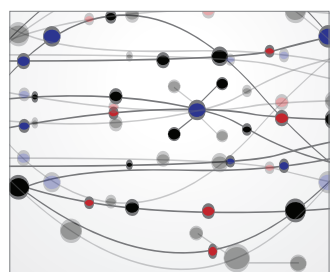

\section{The Scientific} World Journal
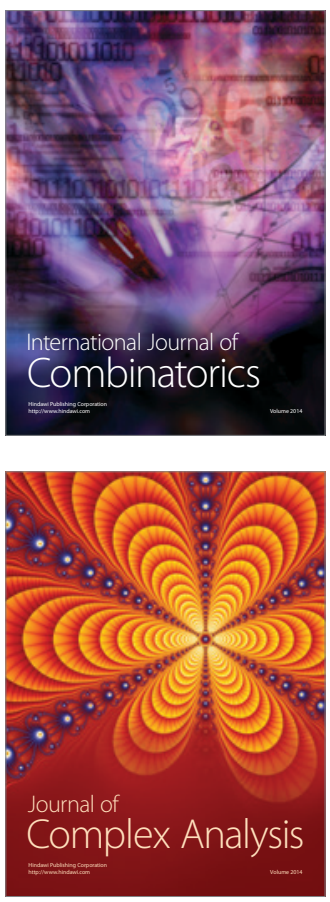

International Journal of

Mathematics and

Mathematical

Sciences
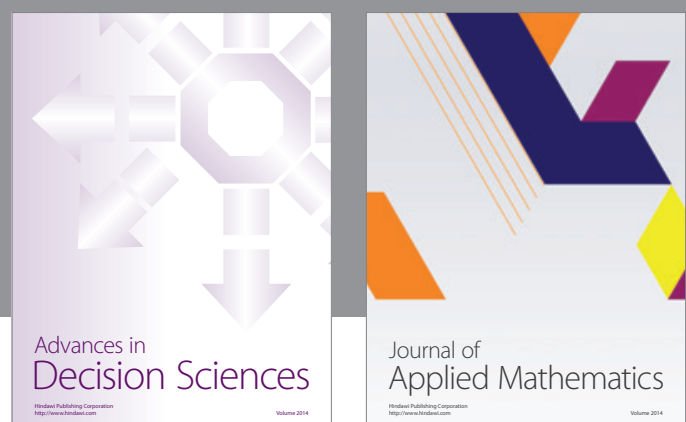

Journal of

Applied Mathematics
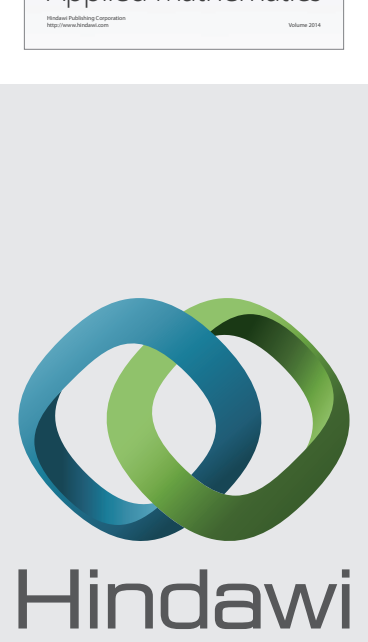

Submit your manuscripts at http://www.hindawi.com
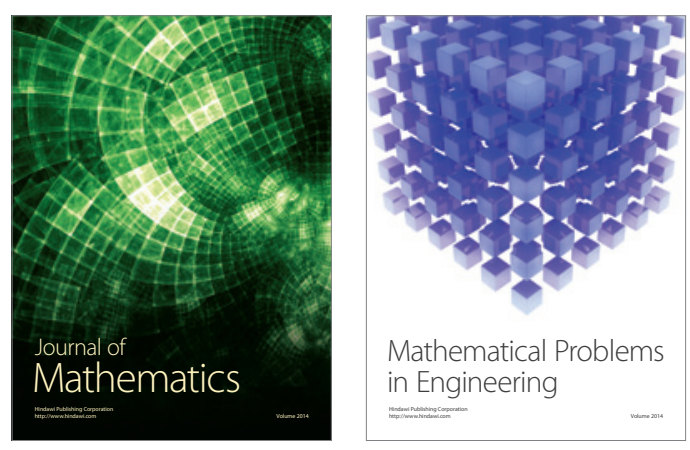

Mathematical Problems in Engineering
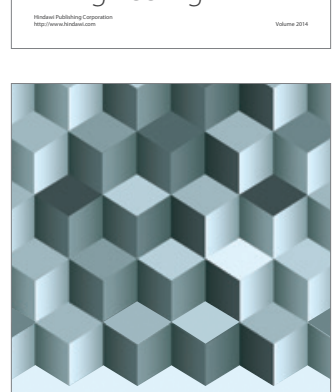

Journal of

Function Spaces
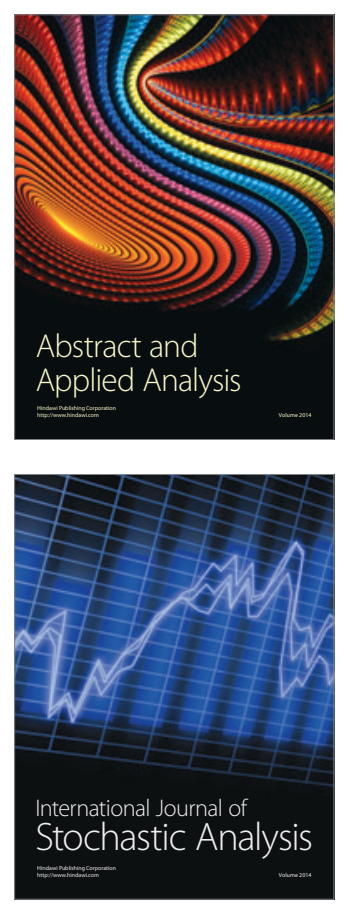

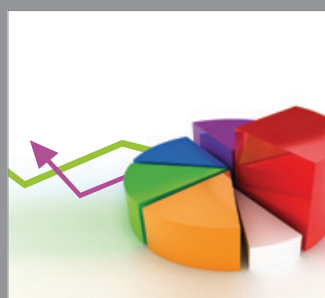

ournal of

Probability and Statistics

Promensencen
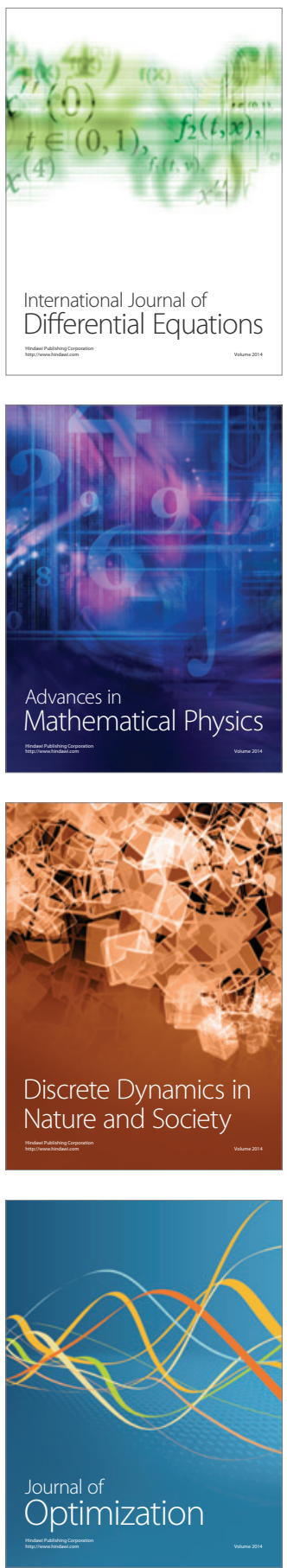